\title{
A New Neutrosophic Approach to Image Denoising
}

\author{
Yanhui Guo $^{1,2}$, H.D. Cheng ${ }^{1,2}$, Yingtao Zhang ${ }^{1}$, Wei Zhao ${ }^{1}$ \\ ${ }^{1}$ School of Computer Science and technology, Harbin Institute of Technology, Harbin, \\ China, 150001 \\ ${ }^{2}$ Department of Computer Science, Utah State University, Logan, UT 84322 U.S.A.
}

\begin{abstract}
A neutrosophic set (NS), a part of neutrosphy theory, studies the origin, nature, and scope of neutralities, as well as their interactions with different ideational spectra. The neutrosophic set is a general formal framework that has been recently proposed. However, the neutrosophic set needs to be specified from a technical point of view. Now, we apply the neutrosophic set into image domain and define some concepts and operators for image denoising.

The image $G$ is transformed into NS domain, which is described using three membership sets: T, I and F. The entropy of the neutrosophic set is defined and employed to evaluate the indeterminancy. A new operation, $\gamma-$ median-filtering operation, is proposed to decrease the set indeterminancy and remove noise. We have conducted experiments on a variety of noisy images using different type of noise with different levels. The experimental results demonstrate that the proposed approach can remove noise automatically and effectively. Especially, it can process not only noisy images with different levels of noise, but also images with different kinds of noise well without knowing the type of the noise, which is the most difficult task for image denoising.
\end{abstract}

Keywords: Image denoising, neutrosophic set, entropy.

\section{Introduction}

Neutrosophy, a branch of philosophy, introduced in [1] as a generalization of dialectics, which studies the origin, nature, and scope of neutralities, as well as their interactions with different ideational spectra.

Neutrosophy theory considers proposition, theory, event, concept, or entity, $<\mathrm{A}>$ is in relation to its opposite, $<$ Anti-A $>$ and the neutrality $<$ Neut-A $>$, which is neither $\langle\mathrm{A}\rangle$ nor $<$ Anti-A $>$. The $<$ Neut-A $>$ and $<$ Anti-A $>$ are referred to as $<$ Non-A $>$. According to this theory, every idea $<\mathrm{A}>$ tends to be neutralized and balanced by $<$ Anti-A $>$ and $<$ Non-A $>$.

In a classical way $<\mathrm{A}>$, $<$ Neut-A $>$ and $<$ Anti-A $>$ are disjoint two by two. In many cases, the borders between notions are vague and imprecise, and it is possible that $<\mathrm{A}\rangle,<$ Neut- $\mathrm{A}>$ and $<$ Anti-A $>$ (and $<$ Non-A $>$ of course) have common parts two by two as well.

The neutrosophy is the basis of the neutrosophic logic, neutrosophic probability, neutrosophic set, and neutrosophic statistics [1].

In neutrosophic set, the indeterminacy is quantified explicitly and the truth-membership, indeterminacymembership and falsity-membership 
are independent. This assumption is very important in many applications such as information fusion in which we combine the data from different sensors. The neutrosophic set is a general formal framework which generalizes the concept of the classic set, fuzzy set [2], interval valued fuzzy set [3], intuitionistic fuzzy set [4], interval valued intuitionistic fuzzy set [5], paraconsistent set [1], dialetheist set [1], paradoxist set [1], and tautological set [1].

Noise is very common in imaging systems which occurs in image acquisition, transmission, etc. Image denoising is one of the most widely investigated topics in image preprocessing, pattern recognition and image vision [6]. Despite that a huge number of approaches has been proposed, denoise algorithms performance mainly depends on a suitable representation to describe the original image information and noise type.

The neutrosohic set had been applied into image processing. [7] proposed a thresholding algorithm based on neutrosophic, which could select the thresholds automatically and effectively. In this paper, a new neutrosphic set approach, NS filter, is proposed for image denoising. First, the image is transformed into the neutrosophic set and a new filtering operation, $\gamma$ median-filtering, is employed to reduce the indetermination degree of the image, which is evaluated by the entropy of the indeterminate subset. After filtering processing, the noise will be removed. The experiments on real images having different kinds of noise with different levels demonstrate that the proposed approach can perform denoising better.

\section{Proposed method}

\subsection{Neutrosphic set}

The definition of a neutrosophic set and its properties are introduced briefly.

Definition 1 (Neutrosophic set) Let $U$ be a universe of discourse, and a neutrosophic set $A$ is included in $U$. An element $x$ in set $A$ is noted as $x(T, I, F) . T, I$ and $F$ are real standard or non-standard sets of $]^{-} 0,1^{+}[$ with sup $T=t_{-}$sup, inf $T=t_{-}$inf , sup $I=i \_$sup , inf $I=i \_i n f$, sup $F=f \_s u p, \quad$ inf $F=f \_$inf and $n \_$sup $=t \_s u p+i \_$sup $+f \_$sup , $n \_i n f=t \_i n f+i \_i n f+f \_i n f . T, I$ and $F$ are called the neutrosophic components.

\subsection{Neutrsophic Image}

Definition 2 (Neutrosophic image): Let $U$ is a universe of discourse, and $W$ is a set of $U$, which is composed by bright pixels. A neutrosophic image $P_{N S}$ is characterized by three membership sets $T, I$ and $F$.

A pixel $P$ in the image is described as $P(T, I, F)$ and belongs to $W$ in the following way: it is $t \%$ true in the bright pixels set, $i \%$ indeterminate, and $f \%$ false, where $t$ varies in $T, i$ varies in $I$, and $f$ varies in $F$.

Then the pixel $P(i, j)$ in the image domain is transformed into the neutrosophic set domain $P_{N S}(i, j)=\{T(i, j), I(i, j), F(i, j)\} \quad$. $T(i, j), I(i, j)$ and $F(i, j)$ are the probabilities belong to white pixels set, indeterminate set and non white pixels set, respectively, which are defined as:

$$
T(i, j)=\frac{\bar{g}(i, j)-\bar{g}_{\min }}{\bar{g}_{\max }-\bar{g}_{\min }}(1)
$$




$$
\begin{gathered}
\bar{g}(i, j)=\frac{1}{w \times w} \sum_{m=i-w / 2}^{i+w / 2} \sum_{n=j-w / 2}^{j+w / 2} g(m, n) \\
I(i, j)=\frac{\delta(i, j)-\delta_{\min }}{\delta_{\max }-\delta_{\min }}(3) \\
\delta(i, j)=a b s(g(i, j)-\bar{g}(i, j)) \\
F(i, j)=1-T(i, j)(5)
\end{gathered}
$$

where $\bar{g}(i, j)$ is the local mean value of the pixels of the window. $\delta(i, j)$ is the absolute value of difference between intensity $g(i, j)$ and its local mean value $\bar{g}(i, j)$.

\subsection{Neutrosophic Image Entropy}

Definition 7 (Neutrosophic image entropy) Neutrosphic entropy of an image is defined as the summation of the entropies of three subset $T, I$ and $F$ :

$$
\begin{gathered}
E n_{N S}=E n_{T}+E n_{I}+E n_{F} \text { (6) } \\
E n_{T}=-\sum_{i=\min \{T\}}^{\max \{T\}} p_{T}(i) \ln p_{T}(i) \\
E n_{I}=-\sum_{i=\min \{I\}}^{\max \{I\}} p_{I}(i) \ln p_{I}(i) \\
E n_{F}=-\sum_{i=\min \{F\}}^{\max \{F\}} p_{F}(i) \ln p_{F}(i)
\end{gathered}
$$

where $E n_{T}, E n_{I}$ and $E n_{F}$ are the entropies of sets $T, I$ and $F$, respectively. $p_{T}(i), p_{I}(i)$ and $p_{F}(i)$ are the probabilities of elements in $T, I$ and $F$, respectively, whose values equal to i.

\section{4. $\gamma$-median-filtering operation}

The values of $I(i, j)$ is employed to measure the indeterminate degree of element $P_{N S}(i, j)$. To make the set $I$ correlated with $T$ and $F$, the changes in $T$ and $F$ influence the distribution of element in $I$ and vary the entropy of $I$.
Definition 8 ( $\gamma$-median-filtering operation): A $\gamma$-median-filtering operation for $P_{N S}, \hat{P}_{N S}(\gamma)$, is defined as:

$$
\begin{gathered}
\hat{P}_{N S}(\gamma)=P(\hat{T}(\gamma), \hat{I}(\gamma), \hat{F}(\gamma))(10) \\
\hat{T}(\gamma)=\left\{\begin{array}{ll}
T & I<\gamma \\
\hat{T}_{\gamma} & I \geq \gamma
\end{array}(11)\right. \\
\hat{T}_{\gamma}(i, j)=\operatorname{median}_{(m, n) \in S_{i j}}\{T(m, n)\}(12) \\
\hat{F}(\gamma)=\left\{\begin{array}{ll}
F & I<\gamma \\
\hat{F}_{\gamma} & I \geq \gamma
\end{array}(13)\right. \\
\hat{F}_{\gamma}(i, j)=\operatorname{median}_{(m, n) \in S_{i j}}\{F(m, n)\}(14) \\
\hat{I}_{\gamma}(i, j)=\frac{\delta_{\hat{T}}(i, j)-\delta_{\hat{T} \min }}{\delta_{\hat{T} \max }-\delta_{\hat{T} \min }}(15) \\
\delta_{\hat{T}}(i, j)=a b s(\hat{T}(i, j)-\hat{\hat{T}}(i, j))(16) \\
\overline{\hat{T}}(i, j)=\frac{1}{w \times w} \sum_{m=i-w / 2}^{i+w / 2} \sum_{n=j-w / 2}^{j+w / 2} \hat{T}(m, n)(17)
\end{gathered}
$$

where $\bar{\delta}_{T}(i, j)$ is the absolute value of difference between intensity $\hat{T}(i, j)$ and its local mean value $\overline{\hat{T}}(i, j)$ at $(i, j)$ after $\gamma$-median-filtering operation.

The new neutrosophic approach to image denoising is described as bellows:

Step 1: Transform the image into NS domain;

Step 2: Use $\gamma$-median-filtering operation on the true subset $T$ to obtain $\hat{T}_{\gamma}$;

Step 3: Compute the entropy of the indeterminate subset $\hat{I}_{\gamma}, E n_{\hat{I}_{\gamma}}(i)$;

Step 4: If $\frac{E n_{\hat{I}_{y}}(i+1)-E n_{\hat{I}_{y}}(i)}{E n_{\hat{I}_{y}}(i)}<\delta$, go to

Step 5;

$$
\text { Else } T=\hat{T}_{\gamma} \text {, go to Step 2; }
$$

Step 5: Transform subset $\hat{T}_{\gamma}$ from the neutrosophic domain into gray level domain. 


\section{Experimental results}

In the experiments, we used 'Lena', to evaluate the performance of the proposed method and compare with two filters: median filter and mean filter. Different kinds of noise with different levels are added into the image.

The performance of the denoising algorithm is measured by the peak-singalto-noise ( $P N S R$ ) [8], which is defined as:

$P S N R=-10 \log \left[\frac{\sum_{r=0}^{H-1} \sum_{c=0}^{W-1}\left(I(r, c)-I_{n}(r, c)\right)^{2}}{H \times W \times 255^{2}}\right]$

where $I(r, c)$ and $I_{n}(r, c)$ represent the intensities of pixel $(r, c)$ in the noise-free images and images after filtering, respectively. The higher the PSNR is, the better the denoising algorithm is.

In first step, the proposed denosing algorithm, NS filter is compared with median filter and mean filter in removing Gaussian noise with different variances. Fig. 1(a) is the original image, and Fig. 1(b) is the image resulted of Fig. 1(a) added with Gaussian noise with 0 mean and 22.5 variance. Figs. 1(c) and 1(d) are the denoising results of median filter and mean filter, respectively. Figs. 1(e) is the result of the proposed method, NS filter.

From the comparison on visual and quantity measures in Fig. 1, we can conclude that the NS denoising method not only removes Gaussian noise properly and achieves best visual result in Fig. 1(e), but also has higher PNSR .



(a)



(b)



(c)

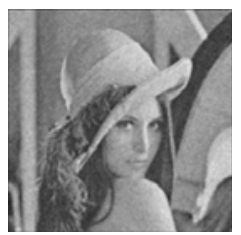

(d)

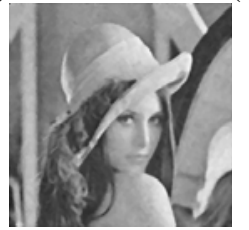

(e)

Fig.1. (a) The original image. (b) The original image with Gaussian noises (mean: 0, standard deviation: 22.5). (c) The image filtered using median filter (PSNR: 25.95).

(d) The image filtered using mean filter (PSNR: 26.25). (e) The image filtered using NS filter (PSNR: 26.95).

Secondly, a series of Lena images with different variances are employed to test the performance of the NS filter, median filter and mean filter. The PSNR values of these results are shown in Fig. 3. The standard deviation value of Gaussian noise added to Lena image changes from 25.5 to 255 . The PSNR values of the images using NS filter, median filter and mean filter vary from 26.95 to $21,25.95$ to 17.44 and 26.25 to 19.82 , respectively. At different noise level, the PSNR values of NS filter is always the highest, which means that NS filter achieve the best performance on Gaussian noise removing.

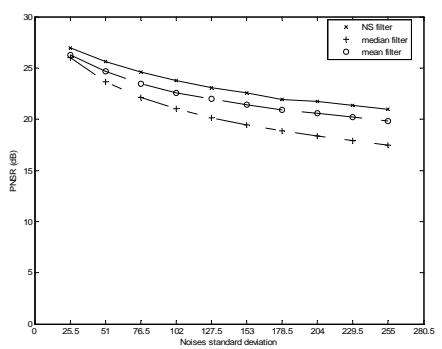

Fig. 2. The comparison of different filters on images with Gaussian noise. $\times$ : NS filter; + : Median filter; $\bigcirc$ : Mean filter. 
Meanwhile, NS filter is employed to remove other types of noise, such as Salt and Peeper noise. In Fig. 3, we can see that the NS filter outperforms median and mean filters on images with Salt and Pepper noise with 20\% noise densities.

Another group of Lena images with Salt and Pepper noise with different noise densities are utilized to remove noises by using the NS filter, median filter and mean filter. The PSNR values of these results are shown in Figure 4. The noise density value changes from $20 \%$ to $75 \%$ on Lena image. The PSNR values of the images using $N S$ filter, median filter and mean filter vary from 28.08 to $17.56,27$ to 11.08 , and 20.72 to 14.98 , respectively. At different noise levels, the PSNR values of NS filter is always the highest, which means that NS filter achieve the best performance on Gaussian noise removing. From Fig. 4, we can see that the NS filter outperforms median and mean filters on images with different noise densities.



(a)

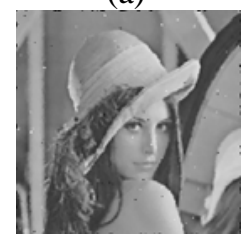

(c)

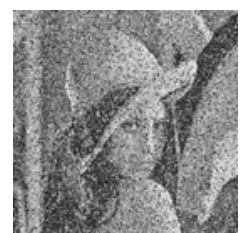

(b)



(d)



(e)

Fig. 3. (a) The Lena image. (b) The image with Salt and Peeper noises (noise density: 0.2). (c) The image filtered using median filter (PSNR: 27.01). (d) The image filtered using mean filter (PSNR: 20.72). (e) The image filtered using NS filter (PSNR: 28.08).

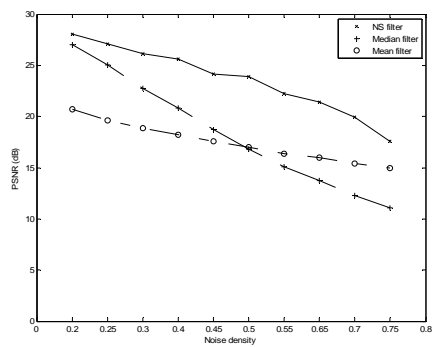

Fig. 4. The comparison of different filters on images with Salt and Pepper noise. $\times$ : NS filter; + : Median filter; $\bigcirc$ : Mean filter.

\section{Conclustions}

In this paper, a novel technique, neutrosophic image denoisng approach, is proposed based neutrsosophic set. The image $G$ is described as a NS set using three membership sets $T, I$ and $F$. The entropy in neutrosophic image domain is defined and employed to evaluate the indetermination. A new filtering operation, $\gamma$-median-filtering operation, is proposed to reduce the set's indetermination and remove the noise in image. The experimental results show that the proposed method can perform better not only on the images with the noise of different kinds levels, but also on the images with different kinds of noise well without knowing the type of the noise, which is the most difficult task for image denoising. The properties of neutrosophic image will achieve more applications in image processing and computer vision.

\section{Acknowledgement}

The work was supported, in part, by Natural Scientific Research Innovation Foundation in Harbin Institute of Technology, Project HIT.NSRIF.2008.48, and 
Natural Science Foundation of China No.60873142 and No. 30670546.

\section{References}

[1] F. Smarandache, A Unifying Field in Logics Neutrosophic Logic. Neutrosophy, Neutrosophic Set, Neutrosophic Probability, third ed: American Research Press, 2003.

[2] L. A. Zadeh, "Fuzzy sets," Inform and control, vol. 8, pp. 338-353, 1965.

[3] I. Turksen, "Interval valued fuzzy sets based on normal forms," Fuzzy Sets and Systems, vol. 20, pp. 191-210, 1986.

[4] K. Atanassov, "Intuitionistic fuzzy sets," Fuzzy Sets and Systems, vol. 20, pp. 87-96, 1986.
[5] K. Atanassov, "More on intuitionistic fuzzy sets," Fuzzy Sets and Systems, vol. 33, pp. 37-46, 1989.

[6] R. C. Gonzalez and R. E. Woods, Digital image processing, 2nd ed: Addison-Wesley Reading, Mass, 1992.

[7] H. D. Cheng and Y. Guo, "A new neutrosophic appraoch to image thresholding," accepted by New Mathematics and Natural Computation, 2008.

[8] G. Y. Chen and B. Kégl, "Image denoising with complex ridgelets," Pattern Recognition, vol. 40, no. 2, pp. 578-585, 2007. 